\title{
The electromyographic activity characteristics of the gluteus medius muscle before and after total hip arthroplasty
}

\author{
KonRAD KoPeĆ ${ }^{1}$, PrZEMYSŁaW BEREZA ${ }^{1 *}$, GRZEGORZ SOBOTA ${ }^{2}$, \\ GRZEGORZ HAJDUK ${ }^{3}$, DAMIAN KUSZ ${ }^{1}$ \\ ${ }^{1}$ Department of Orthopedics and Traumatology, Faculty of Medical Sciences in Katowice, \\ Medical University of Silesia, Katowice, Poland. \\ ${ }^{2}$ Institute of Sport, Department of Human Motor Behaviour, \\ The Jerzy Kukuczka Academy of Physical Education, Katowice, Poland. \\ ${ }^{3}$ Department of Functional Diagnostics and Sports Medicine, \\ The Jerzy Kukuczka Academy of Physical Education, Katowice, Poland.
}

\begin{abstract}
Purpose: The clinical outcomes of total hip arthroplasty are influenced by the correct muscle function that determines good, longterm and proper function of the artificial joint. The aim of the study was to analyze the electromyographic activity of the gluteus medius muscle in patients with hip osteoarthritis and after arthroplasty in various static weight bearing conditions, both on the affected and contralateral side. Methods: The prospective study involved 70 patients qualified for hip replacement. Patients underwent a surface electromyography of the gluteus medius muscle which involved the Trendelenburg test. The normalized results were obtained for both hips, preoperatively and 6 months after arthroplasty. Results: The only muscle activity differences were found at a full load condition of lower limb. In the preoperative assessment, the activity of the gluteus medius muscle was greater on the side qualified for surgery. After arthroplasty and the rehabilitation period, the muscle activity on the operated side decreased and significantly increased on the contralateral side. Detailed analysis of the contralateral side revealed relationship with osteoarthritis. Previous hip arthroplasty of that side resulted in lower muscle activity, similar to fully functional joints. Conclusion: The activity characteristics of the gluteus medius muscle vary depending on the condition of the joint, and the characteristics change as a result of the surgical procedure performed on both the operated and contralateral sides. These dependencies should be taken into account in the rehabilitation process, especially at the side opposite to the operated one.
\end{abstract}

Key words: osteoarthritis, total hip replacement, gluteus medius activity, hip joint

\section{Introduction}

Hip osteoarthritis (OA) significantly disturbs the complicated biomechanical system of the hip joint. The goal of surgical treatment is the hip joint reconstruction using implants [11], [17]. Biomechanical reconstruction of the musculoskeletal system is significantly influenced by the correct muscle function that determines good, long-term and correct function of the artificial joint. The subject of the study was to assess the function of the gluteus medius (GM) muscle, one of the most important muscle conditioning the proper biomechanics of the hip joint [3], [20]. The Trendelenburg test is a functional test that was included in the study protocol. It measures the frontal plane tilt angle of the pelvis during single leg stance. A negative Trendelenburg test reflects desirable hip abductor muscle function and stable gait, whereas a positive Trendelenburg test is associated with hip abductor dysfunction and gait disturbances [8]. This test is now used worldwide as a method for evaluating hip abductor

\footnotetext{
* Corresponding author: Przemysław Bereza, ul. Ziołowa 45/47, 40-635 Katowice, Poland. E-mail address: pbereza@sum.edu.pl Received: November 17th, 2020

Accepted for publication: February 11th, 2021
} 
function after total hip arthroplasty (THA) [19]. In addition, electromyographic examination has been used in the analysis of hip abduction muscle sufficiency because it made it possible to measure timing and relative intensity of muscle activation. The electromyography (EMG) signals of GM muscle were analyzed in patients with hip OA and in patients after total hip arthroplasty [10]. Undoubtedly, disease of one hip joint has a significant impact on the entire musculoskeletal system, especially on the contralateral joint [9]. Most of the available publications concern the functional evaluation of the operated hip joint both before and after THA. The subject of this study was also the opposite joint, regardless of its clinical condition [10], [12], [13]. Assuming the proper biomechanical reconstruction of the hip OA, we expect an improvement in muscle function of both hips. The aim of this study was to analyze the electromyographic activity of the GM muscle in patients with hip OA before and after THA in various static weight bearing conditions on both affected and contralateral hips.

\section{Materials and methods}

\section{Participants}

70 patients were qualified to this prospective study. The patients were operated in the Department of Orthopedics and Traumatology of the Medical University of Silesia in Katowice, Faculty of Medical Sciences in Katowice. The research were conducted between 2015-2019. Due to different technical and medical reason only 64 patients finished evaluation (characteristic of subjects in Table 1).

The main inclusion criterion was primary hip OA, and the exclusion criteria included: 1) secondary hip OA, excluding the low-grade developmental dysplasia of the hip joint (Crowe > 1) [23]; 2) performed surgical treatment of the hip prior to qualification for arthroplasty (arthroscopy, removal of the femoroacetabular impingement, osteotomy and others); 3) shortening of a leg qualified for arthroplasty $>3 \mathrm{~cm}$; 4) history of severe leg injuries followed by surgical treatment or prolonged immobilization; 5) neurological disorders, including paresis and radiculopathies; 6) malformations of the lower limbs; 7) performed arthroplasty of another lower limb joint (knee or ankle joint); 8) performed arthrodesis or lower limb osteotomy; 9) THA performed in another department; 10) time from arthroplasty less than 6 months or longer than 8 months; 11) rehabilitation conducted contrary to the protocol requested in the study; 12) lack of patient consent for participation in the study.

Because of the polyarticular and heterogenic nature of OA, the patients qualified for the study constituted a heterogeneous group. Some of the patients qualified for THA were also diagnosed with contralateral hip OA and qualified for conservative treatment or contralateral THA at a later time. Another group of patients included individuals with unilateral hip OA and normal contralateral hip. The third group consisted of patients qualified for hip replacement after performed previously contralateral THA. Due to the aforementioned heterogeneity of patients, it was decided to treat each individual joint as a separate entity and subject of the analysis. The study did not take sided dominance into account. Individual 128 hips were assigned to the following groups:

- O - hip OA qualified for arthroplasty (examination before, $N=38$ ) and after performed THA (examination after, $N=64$ ),

- C - contralateral joint to operated hip (before, $N=38 ;$ after, $N=64$ ),

and in the detailed condition of the hip:

- $\mathbf{C H}$ - healthy hip, contralateral to operated hip (before, $N=14$; after, $N=28$ ),

- $\mathbf{C E}$ - previously performed THA, contralateral to operated hip (before, $N=12$; after, $N=14$ ),

- $\mathbf{C C}$ - hip joint affected by OA, contralateral to operated hip (before, $N=12$; after, $N=22$ ).

The subjects were informed about the study design and signed informed consent to the study. The research was approved by the Bioethics Committee of the Medical University of Silesia in Katowice.

Table 1. The subjects' body mass and age separate for evaluation time (before and after surgery) and sex

\begin{tabular}{|l|c|c|c|c|c|c|c|}
\hline \multirow{2}{*}{ Period } & \multirow{2}{*}{$N$} & \multicolumn{2}{|c|}{ Body mass $[\mathrm{kg}](\mathrm{mean} \pm \mathrm{STD})$} & \multicolumn{3}{c|}{ Age [years] (mean \pm STD) } \\
\cline { 3 - 8 } & & $\mathrm{W}$ & $\mathrm{M}$ & Total & $\mathrm{W}$ & $\mathrm{M}$ & Total \\
\hline Before & $\begin{array}{c}38 \\
(24 \mathrm{~W}, 14 \mathrm{M})\end{array}$ & $64.3 \pm 11.2$ & $85.1 \pm 10.7$ & $73.1 \pm 14.4$ & $62.8 \pm 14.8$ & $55.2 \pm 13.6$ & $59.5 \pm 14.5$ \\
\hline After & $\begin{array}{c}64 \\
(40 \mathrm{~W}, 24 \mathrm{M})\end{array}$ & $68.9 \pm 10.8$ & $82.3 \pm 13.1$ & $74.5 \pm 13.2$ & $63.4 \pm 14.2$ & $56.1 \pm 12.8$ & $61.2 \pm 13.3$ \\
\hline
\end{tabular}

$N$ - group size, $\mathrm{W}$ - women, $\mathrm{M}$ - men, STD - standard deviation. 
Experimental procedures and instruments. The study used surface EMG in a bipolar system (two active receiving electrodes) with a TeleMyo 400 recorder (Noraxon, USA). The recorder is a portable fourchannel recorder with a $1000 \mathrm{~Hz}$ signal sampling frequency. Dedicated MyoResearch XP Master Edition software (Noraxon, USA) was used to support the recorder, signal processing and data archiving. The principles of patient preparation for EMG, electrode placement and the entire study were in accordance with the procedure SENIAM (Surface Electromyography for the Non-Invasive Assessment of Muscles) and ISEK (International Society of Electromyography and Kinesiology) [2], [18]. Active electrodes were placed for the GM at $1 / 2$ distance measured between the iliac crest and the greater femoral trochanter along the muscle course, at a distance of about $15-20 \mathrm{~mm}$.

The electromyographic activity of the GM of both hips was measured on the test stand for normalizing the results to the maximum voluntary isometric contraction (MVIC) [4]. The measuring stand consisted of an examination bed with a movable resistor. The MVIC test was performed lying on side. An adjustable abductor resistor was placed at the height of the patient's fibula head, and the resistance was set at a height equal to the width of the pelvis. Patients sought to perform hip abduction (repeated 5 times) with their maximum commitment to evaluate the MVIC. Next, the Trendelenburg test were performed and muscle activity were measured for a support limb (loaded with around $80 \%$ of body weight - Load A), and for a lifted leg (loaded by leg weight, around $20 \%$ of body weight - Load B). EMG recording was also performed after changing the weight bearing limbs. The mean values of normalized bioelectrical activity of the GM (mGM) were submitted for statistical analysis.

Patients were then operated. The cementless press-fit arthroplasties were performed by one team of surgeons. From the first day after operation patients were encouraged to get out of bed, walk with frame and then with crutches. Physical rehabilitation lasted up to 7-10 days after surgery. During early postoperative home rehabilitation patients implemented a rehabilitation program and walked with two crutches non weight bearing for the next 4 weeks. For the following 4 weeks patients walked with partial weight bearing with one crutch held in the opposite hand to the operated side. All patients underwent hospital rehabilitation after 12 weeks from operation, which lasted 3 weeks. Within about 6 months after THA patients were referred for the next surface EMG of GM of both hips, according to the previous scheme.
Statistical analysis. Two-factor analysis of variance was used to determine the effect of hip condition and load value of lower limb onto muscle activity of GM. A significance level of $\alpha=0.05$ was assumed for all tests.

Due to non-compliance with the distribution normality condition by all compared groups and frequent right-skewed distribution, logarithmic transformation of the data was performed. As a result, the normal distribution confirmed by the Shapiro-Wilk test and homogeneity of variance confirmed by the Leven test was obtained [22].

\section{Results}

The median values (and first-third quartiles) of muscle activity (calculated as \% MVIC) due to the hip condition, limb load and period of observation are presented in Table 2.

The first step was to analyze the differences between bioelectric activity within the operated "O" and contralateral joints "C" according to the load limb condition before (Fig. 1) and after THA (Fig. 2).

The analysis of results before surgery indicated significant higher level of muscle activity for tests with full weight bearing $(\mathrm{F}(1.44)=13.8 ; p<0.005)$, which could be expected. However, it was surprising that for the group of contralateral joints there was no significant differentiation between the load conditions. Also, with the low limb load (only leg weight, "Load B"), similar activity level was recorded for the group $\mathrm{O}$ and $\mathrm{C}$. The difference between groups during full load (body weight, "Load A") pointed out to higher muscle activity of side qualified to the hip surgery (group $\mathrm{O}$ ), but was not confirmed statistically.

The results after surgery (Fig. 2) showed that the load factor (A/B load) significantly affects the higher values of muscle activity while loading the limb of the whole-body weight $(\mathrm{F}(1.120)=79.56 ; p<0.0001)$, as before treatment. Also, low load condition ("Load B") showed results similar as in the first evaluation. We observed opposite characteristic during full load conditions, because of decreased muscle activity on operated side (from 40 to $31 \%$, group O) and increased on non-operated side (from 25 to $41 \%$, group C).

Due to the fact that joints opposite to those affected by OA were a range of clinical state, from healthy joints, through OA, to cases after arthroplasty, it was decided to deepen this analysis, considering the condition of the contralateral joint in detail. 
Table 2. Median value and Quartiles (first - third) of mean muscle activity (\% MVIC) in the Trendelenburg test separate for load condition (A and B), hip condition

( $\mathrm{O}$ and $\mathrm{C}$ side; $\mathrm{O}, \mathrm{CC}, \mathrm{CE}$ and $\mathrm{CH}$ group) and evaluation time (before and after surgery)

\begin{tabular}{|c|c|c|c|c|c|c|c|c|}
\hline Load & Side & Period & $\mathrm{N}$ & $\begin{array}{l}\text { Median } \\
\text { (Q1-Q3) }\end{array}$ & Group & Period & $\mathrm{N}$ & $\begin{array}{l}\text { Median } \\
\text { (Q1-Q3) }\end{array}$ \\
\hline \multirow{8}{*}{ Test A } & \multirow{2}{*}{$\mathrm{O}$} & Before & 38 & \multicolumn{5}{|l|}{$36.2(21-54)$} \\
\hline & & After & 64 & \multicolumn{5}{|l|}{$28.2(23-40)$} \\
\hline & \multirow{6}{*}{$\mathrm{C}$} & \multirow{3}{*}{ Before } & \multirow{3}{*}{38} & \multirow{3}{*}{$\begin{array}{c}26.3 \\
(16-35)\end{array}$} & \multirow{2}{*}{$\mathrm{CC}$} & Before & 12 & $28.9(25-38)$ \\
\hline & & & & & & After & 22 & $43.8(35-73)$ \\
\hline & & & & & \multirow{2}{*}{$\mathrm{CE}$} & Before & 12 & $17.6(14-26)$ \\
\hline & & \multirow{3}{*}{ After } & \multirow{3}{*}{64} & \multirow{3}{*}{$\begin{array}{c}37.7 \\
(23-52)\end{array}$} & & After & 14 & $23.0(18-35)$ \\
\hline & & & & & \multirow{2}{*}{$\mathrm{CH}$} & Before & 14 & $29.4(12-39)$ \\
\hline & & & & & & After & 28 & $37.8(19-52)$ \\
\hline \multirow{8}{*}{ Test B } & \multirow{2}{*}{$\mathrm{O}$} & Before & 38 & \multicolumn{5}{|l|}{$14.6(7-19)$} \\
\hline & & After & 64 & \multicolumn{5}{|l|}{$11.4(6-18)$} \\
\hline & \multirow{6}{*}{$\mathrm{C}$} & \multirow{3}{*}{ Before } & \multirow{3}{*}{38} & \multirow{3}{*}{$\begin{array}{c}12.4 \\
(6-16)\end{array}$} & \multirow{2}{*}{$\mathrm{CC}$} & Before & 12 & $12.7(10-17)$ \\
\hline & & & & & & After & 22 & $13.0(8-20)$ \\
\hline & & & & & \multirow{2}{*}{$\mathrm{CE}$} & Before & 12 & $9.6(5-21)$ \\
\hline & & \multirow{3}{*}{ After } & \multirow{3}{*}{64} & \multirow{3}{*}{$\begin{array}{c}12.3 \\
(7-18)\end{array}$} & & After & 14 & $12.3(4-25)$ \\
\hline & & & & & \multirow{2}{*}{$\mathrm{CH}$} & Before & 14 & $12.4(6-16)$ \\
\hline & & & & & & After & 28 & $10.5(7-16)$ \\
\hline
\end{tabular}

$N$ - group size, Q1 - first quartile, Q3 - third quartile.

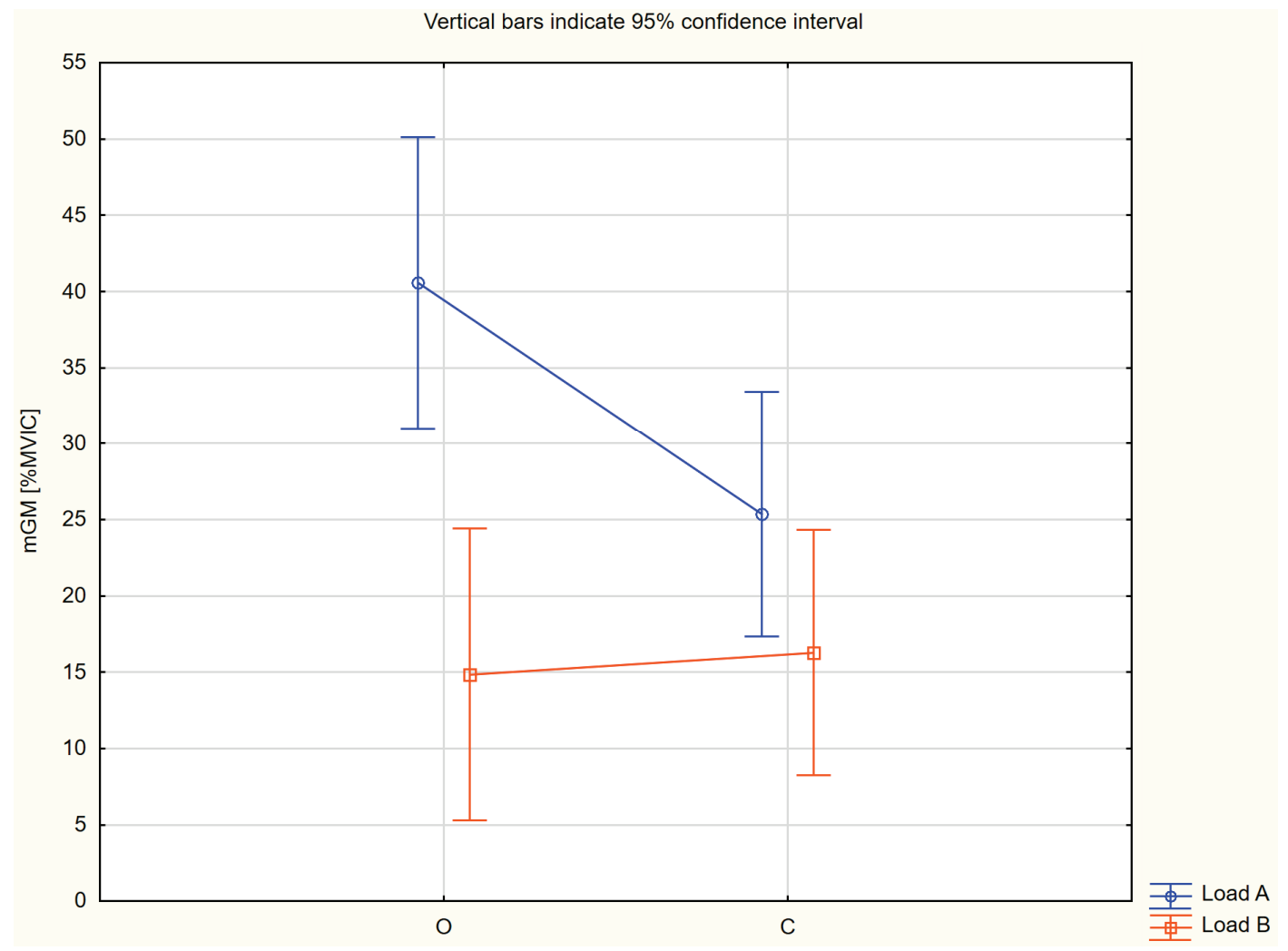

Fig. 1. Mean normalized GM activity [\%MVIC] of the operated $(\mathrm{O})$ and contralateral $(\mathrm{C})$ sides for Load A and B condition before arthroplasty

The mean values before surgery indicate that joints qualified for surgery $(\mathrm{O})$ as well as opposing joints affected by OA (CC) present the highest values in full weight bearing tests (Load A, Fig. 3). The cases of healthy joints and those previously operated showed lower activity (CE and CH, Load A, Fig. 3), slightly higher than in the case of low weight bearing. 
Vertical bars indicate $95 \%$ confidence interval

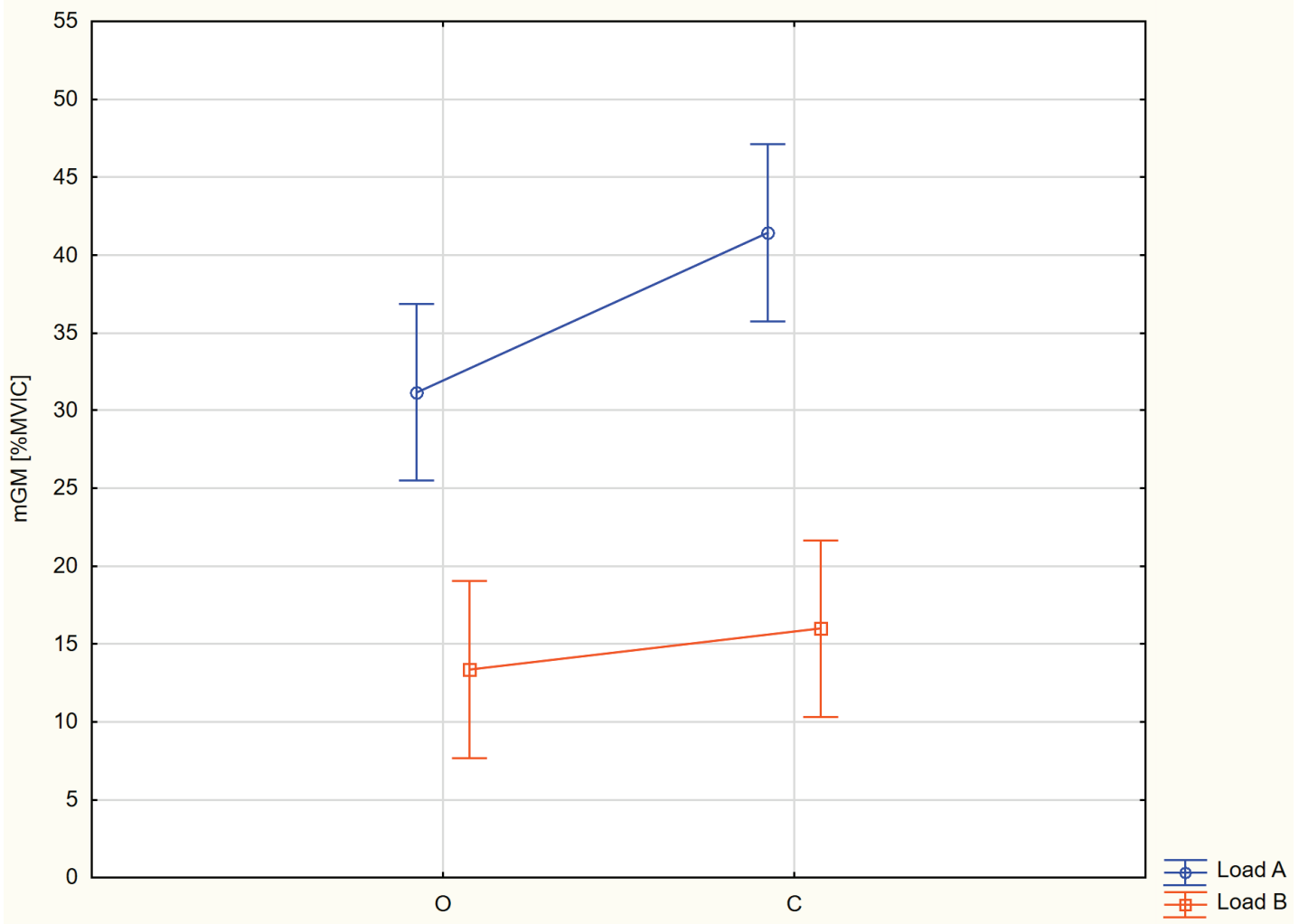

Fig. 2. Mean normalized GM activity [\%MVIC] of the operated $(\mathrm{O})$ and contralateral $(\mathrm{C})$ sides for Load A and B condition after arthroplasty

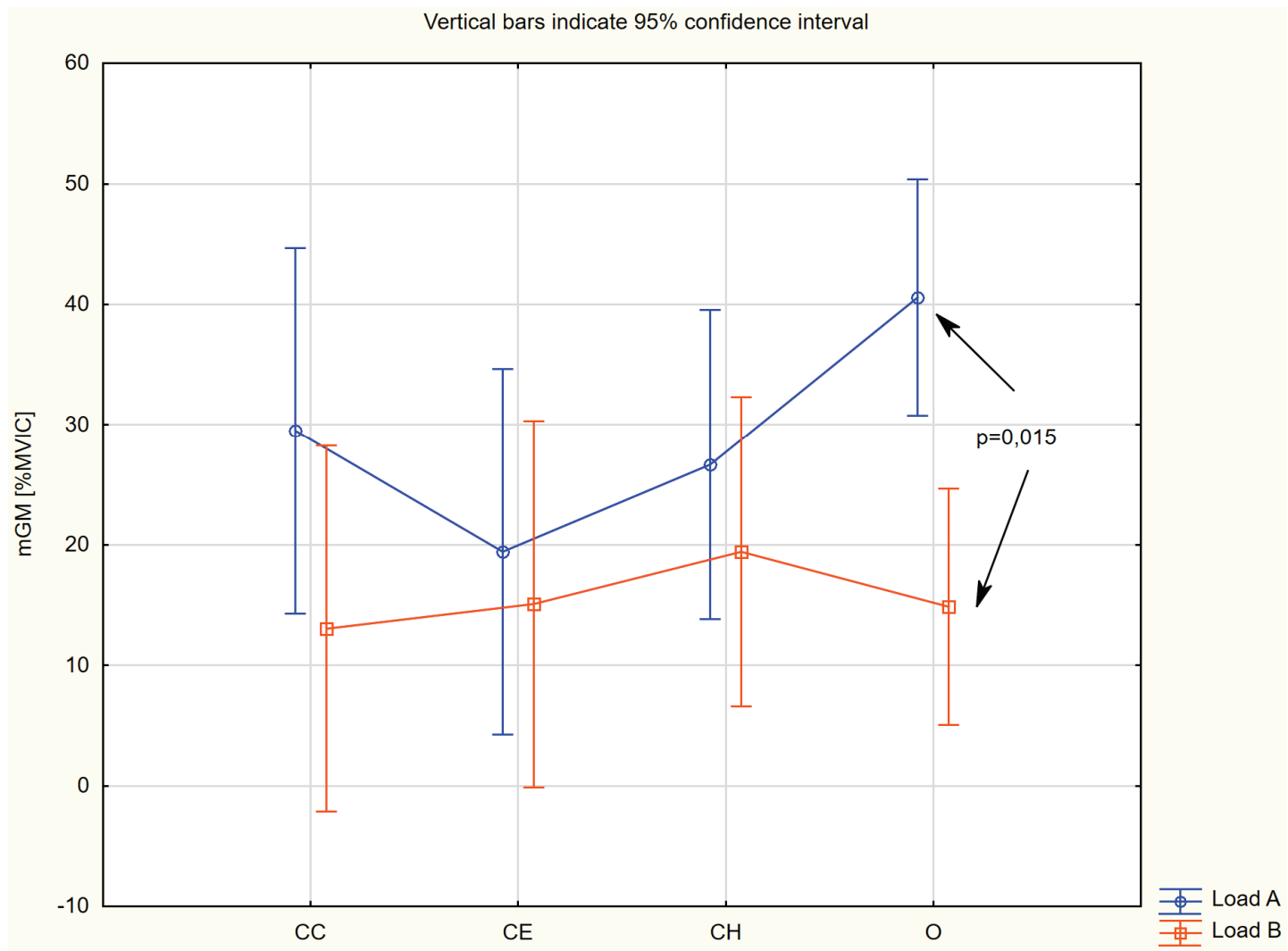

Fig. 3. Mean normalized GM activity [\%MVIC] for detailed classification of hip joints for Load A and B condition before arthroplasty 


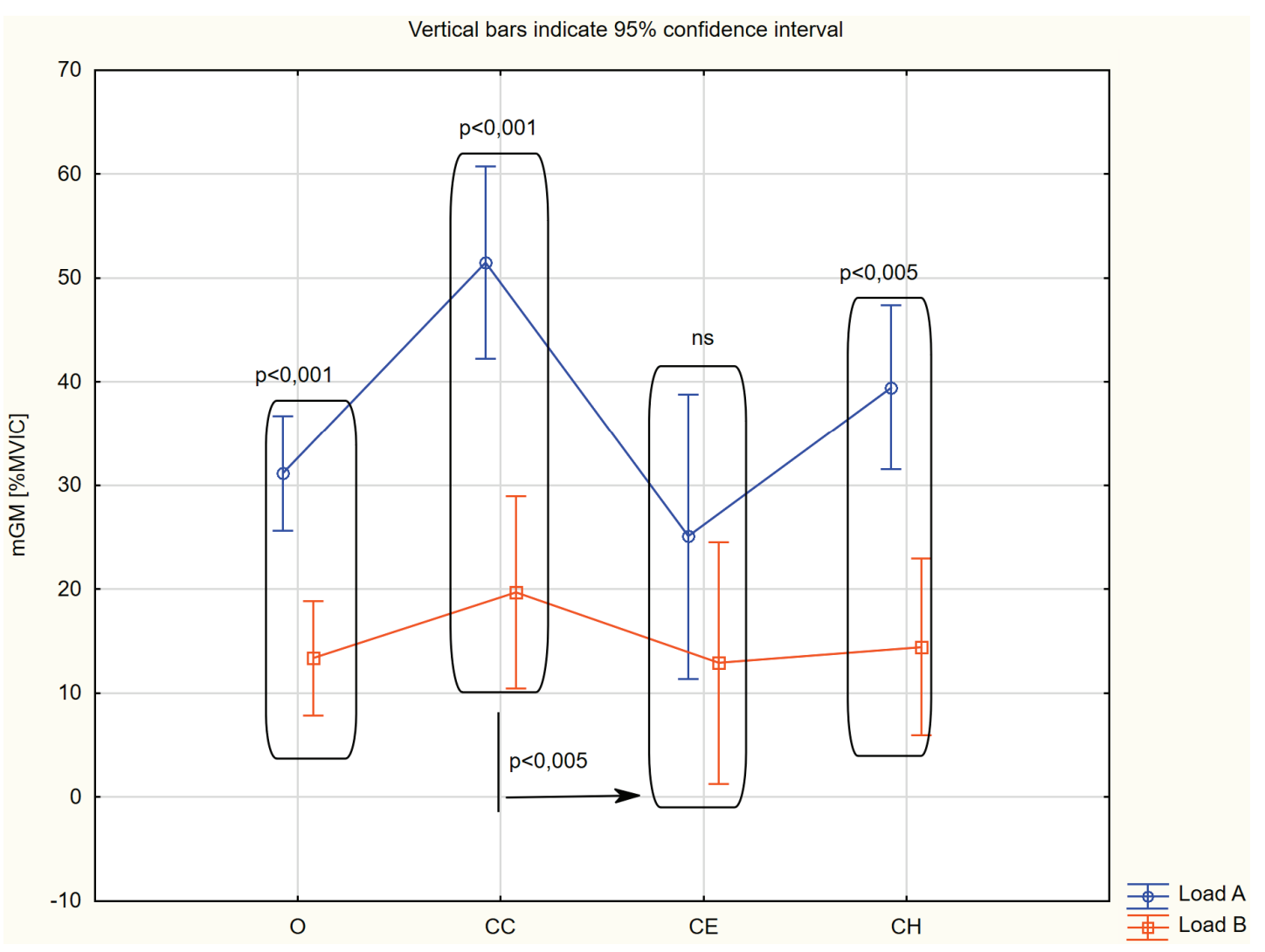

Fig. 4. Mean normalized GM activity [\%MVIC] for detailed classification of hip joints for Load A and B condition after arthroplasty

The lack of differentiation based on the type of load observed previously (Fig. 1) for whole group C, now has better view of intra group variability (Fig. 3). The analysis of variance indicates only the significant factor of the type of load (F $(1.40)=6.69 ; p<0.05)$, while the post hoc analysis has only one significant difference - a higher mean GM muscle activity for operated side (group $\mathrm{O}, p=0.015$ ).

The significant effect of leg load condition at second evaluation pointed on higher muscle activity at "Load A" (F (1.116) = 59.27; $p<0.0001)$, but post hoc tests showed no differentiation only in the CE group due to the size of the load (Fig. 4).

However, it turned out that the assignment of hips to detailed categories significantly differentiates the results obtained $(\mathrm{F}(3.116)=3.01 ; p=0.033)$, and a detailed analysis indicates significantly higher values of muscle activity in the CC group compared to the CE group ( $p=0.048$ ). For a full weight bearing the joints left for conservative treatment or subsequent intervention obtains the highest activity, while operated joints (at this treatment or previously) present a level similar to healthy joints. For "Load B" conditions no differences were found regardless of hip condition.

\section{Discussion}

An increased muscle activity of the affected hip was noticed in static tests with a full weight bearing condition in the initial examination, although not statistically confirmed. Under low weight bearing conditions a symmetrical distribution of muscle activity was noticed in both hips. The arthroplasty procedure approximates the characteristics of the mean bioelectrical activity of both, the operated and contralateral hip joints in static test conditions and emphasizes the diversity in the muscle tension due to the state of weight bearing. After arthroplasty, the GM muscle opposite to the operated hip, affected by OA, revealed a higher bioelectric activity.

Osteoarthritis is often a polyarticular disease. Patients qualified for arthroplasty have advanced hip dysfunction what could result from long conservative treatment. This dysfunction affects the structures of the entire joint and muscular-ligament system, thus affecting on limitation of limb function and gait pathology. Population studies indicate that approximately $35 \%$ of young patients who have undergone THA require additional follow-up visits during early rehabilitation 
period what is associated with GM failure. It was proved that the correct strength of the abductors returns one year after surgery [12]. Surgery treatment is considered to be terminated when painless gait without orthopedic supports is observed, forgetting that the strength of the abductors increases by only $50 \%$ in 6 months after THA and their dysfunction is recorded electromyographically even 2 years after surgery. The success of a quick return to full weighting of the operated limb without crutches is criticized by many authors, due to the abductors insufficiency which are unable to stabilize the pelvis in such a short time. This failure may lead to joint instability and be the cause of early and late complications of arthroplasty. Hence the role of research on the function of muscle abductors both before and after surgery as well as extended rehabilitation protocols focused on this motor unit [10].

The subject of many studies was muscle activity during gait. Hip abductors are relatively weak muscles of the lower limb, despite their important role in pelvic stabilization. The maximum values of the abductors moments of strength are smaller than the moments of the hip flexors or extensors, although higher than the rotators muscle. Researchers analyzed both physiological gait in healthy individuals and various gait pathologies [7]. During the normal gait each muscle group exhibits their activity. The GM is the most active in the support phase and in addition at the end of the swing phase.

Undoubtedly, greater activity of the GM is observed during full weight bearing (Load A) compared to partial weight bearing with the load of raised limb (Load B). This relationship has been confirmed in the Trendelenburg test for both limbs, affected by OA and opposite, before (Fig. 1; F $(1.44)=13.8 ; p<0.005$ ) and after (Fig. 2, F $(1.120)=79.56 ; p<0.0001$ ) surgery. Similar relationship in healthy people was demonstrated by other authors by electromyographic analysis of 22 exercises activating gluteus muscles where the "pelvic drop" test showed activity at the level of $58.43 \%$ MVIC which placed this exercise on the 11th position in terms of GM activity [16], [20]. It was also shown that for full weight bearing conditions there is a trend towards asymmetric muscle activity (Fig. 1), the greatest on the side with OA (mean 40.5\% MVIC) in relation to the contralateral joint (mean 25\% MVIC). This asymmetry is reduced after THA (Fig. 2) and the level of activity is inverse (lower on the operated side). Although GM in hip affected by OA is inefficient and certainly weaker than healthy, it generates greater electromyographic signals.

Imaging studies additionally confirm the partial atrophy of both superficial and deep muscles in the region of affected hip [6]. In this study muscular atrophy was not assessed, although it was expected in OA hips. Higher EMG activity of muscles around the affected joints was also received by other authors analyzing GM in hip OA as well as quadriceps muscle in knee OA [3], [16], [21]. Several studies try to explain this phenomenon. The hypothesis that patients with hip OA activate muscle more due to faster movements is not confirmed in gait laboratory tests, because they show a significantly lower gait speed than healthy people. Definitely the need to keep the pelvis horizontally in the frontal plane, thus, maintaining the right center of gravity forces greater abductors' activity during standing. The dependence of the osteoarticular system on the musculoskeletal unit is confirmed in many pathologies of the musculoskeletal system. However, the question should be asked whether the primary problem concerns the osteoarticular system and results in muscular dysfunction, or is the opposite situation, and muscular dysfunction predispose to the development of joint disease? Our study, unfortunately, does not explain the order of events, but it undoubtedly confirms the relationship of these two systems [15]. It is possible that the pathology of both systems coexists, and the central nervous system generates more neuromuscular connections by recruiting more motor units to strengthen muscle strength enough to make the move [14]. Advanced OA is also associated with significant pain that stimulates increased muscle tension. In this study a detailed analysis of contralateral muscle activity has been performed. It was shown that for full weight bearing (Load A, Figs. 3, 4), the joint left for conservative treatment or for subsequent intervention obtained the highest activity, while the joints after THA presented an activity level similar to healthy joints. Low weight bearing tests (Load B, Figs. 1-4) showed no differentiation in activity regardless of joint condition. Perhaps, it was reason why it was difficult to show differences in muscle activity in healthy and pathologically affected joints with other low weight bearing tests. Therefore, it seems that the proposed schematic of full (Load A) and partial (Load B) weight bearing confirms the usefulness of the Trendelenburg test in further preoperative EMG signals of GM for hips qualified for THA and in postoperative period, as well as contralateral hips in various clinical conditions. Perhaps, the time of the postoperative examination is important for these results. Significant improvement in the general condition of patients, pain relief, physical disability reduction, as well as improvement of mood and return to social life are observed 3 months after surgery [1]. However, some authors indicate a postoperative period of six months as 
the most important for comprehensive rehabilitation, although gait speed and symmetry of gait improves up to a year after surgery [9]. Our observations indicate an improvement in gait parameters 6 month after operation, although it did not match the values of healthy people [13]. This period allows for the restoration of proper mobility of the operated hip joint, however, improper kinematic of the opposite limb is still observed, especially the knee joint. The correlation and impact of joint surgery on the entire musculoskeletal system and the resulting gait pathologies are commonly described in the literature and do not only apply to arthroplasties [5].

The GM muscle activity on the non-operated hip increases in the group with bilateral OA (CC). An increase in EMG activity could be associated with a full weight bearing of opposite limb, which was qualified for arthroplasty later. However, all examined patients walked with full weight bearing without orthopedic support in postoperative assessment. This study did not include proprioceptive analysis, however, because of changes in the neuromuscular activity of the operated hip, abnormalities of the opposite hip proprioception and the resulting differences in EMG activity of a clinically better joint should be expected. Similar results were obtained in Dwyer's study, which states increased GM electromyographic signals of both hips in patients with advanced unilateral hip OA [3].

\section{Limitations}

Firstly, due to the difficulty in obtaining homogeneous research material, not only in terms of the hip joint condition, but also other factors characterizing the examined patients, individual hip joints were identified as independent units. For this reason, the popular repeated measurement scheme cannot be used in statistical analysis, since not all joints assessed before surgery were also examined after surgery and vice versa. Some people qualified for surgery were disqualified from the study because of anesthesia reasons, others did not comply with the obligation of a follow-up examination. Due to the above-mentioned conditions, it was decided to perform test statistics for independent measurements. Secondly, the small sample size, especially in the $\mathrm{CC}$ and $\mathrm{CE}$ subgroup, probably does not allow to indicate other statistically significant differences confirming the trends of mean values. However, the origin of the study group from one center, the standardized method of treatment and rehabilitation gives the possibility of objective analysis of the studied parameters.

\section{Conclusions}

The analysis of the obtained results of abductors activity after hip replacement emphasizes the diversity in muscle activity resulting from the state of weight bearing, and reveals a higher bioelectric activity of abductor muscle opposite to the operated hip, affected by osteoarthritis. The obtained findings take notice of postoperative rehabilitation protocols, which should be differentiated relatively to the clinical condition of the non-operated limb. It seems reasonable to extend the rehabilitation protocol of this group of patients to nonoperated limb, especially with regard to pelvic stabilization and efficiency of the GM muscle [12]. Increased post-operative GM electromyographic activity on the non-operated side inclines to detailed clinical control. Prolonged waiting time for potential surgical treatment of affected, opposite hip may result in a worse functional outcome of abductors. There is a need for further research to explain whether unilateral OA may be the cause of the progression towards OA of opposite hip.

\section{Acknowledgements}

We would like to thank all members of the Department of Orthopedics and Traumatology who help us to conduct the research and discuss the results. This study received funding from the Medical University of Silesia in Katowice (KNW-1-097/08 and KNW-1-108/N/9/K).

\section{References}

[1] Davis A.M., Perruccio A.V., Ibrahim S., HogG-Johnson S., Wong R., Streiner D.L., Beaton D.E., Côté P., Gignac M.A., FlanNery J., SCHEMITSCH E., MAHOMED N.N., BADlEy E.M., The trajectory of recovery and the inter-relationships of symptoms, activity and participation in the first year following total hip and knee replacement, Osteoarthritis Cartilage, 2011, 19, 1413-1421.

[2] De Luca C.J., Adam A., Wotiz R., Gilmore L.D., Nawab S.H., Decomposition of surface EMG signals, J. Neurophysiol., 2006, 96, 1646-1657.

[3] Dwyer M.K., Stafford K., Mattacola C.G., Uhl T.L., GIORDANI M., Comparison of gluteus medius muscle activity during functional tasks in individuals with and without osteoarthritis of the hip joint, Clin. Biomech., 2013, 28, 757-761.

[4] French H.P., Huang X., Cummiskey A., Meldrum D., MALONE A., Normalisation method can affect gluteus medius electromyography results during weight bearing exercises in people with hip osteoarthritis (OA): a case control study, Gait Posture, 2015, 41, 470-475.

[5] Foucher K.C., Wimmer M.A., Contralateral hip and knee gait biomechanics are unchanged by total hip replacement for unilateral hip osteoarthritis, Gait Posture, 2012, 35, 61-65. 
[6] Grimaldi A., Richardson C., Stanton W., Durbridge G., DONNELLY W., HIDES J., The association between degenerative hip joint pathology and size of the gluteus medius, gluteus minimus and piriformis muscles, Man. Ther., 2009, 14, 605-610.

[7] Hajduk G., Nowak K., Sobota G., Kusz D., Kopeć K., BŁASZCZAK E., CIELIŃSKI Ł., BACIK B., Kinematic gait parameters changes in patients after total knee arthroplasty. Comparison between cruciate-retaining and posterior-substituting design, Acta Bioeng. Biomech., 2016, 18, 137-142.

[8] Hardcastle P., NADE S., The significance of the Trendelenburg test, J. Bone Jt. Surg., 1985, 67, 741-746.

[9] Hodt-Billington C., Helbostad J.L., VervaAt W., Rognsvåg T., Moe-NilsSen R., Criteria of gait asymmetry in patients with hip osteoarthritis, Physiother. Theory Pract., 2012, 28, 134-141.

[10] Jacobs C.A., Lewis M., Bolgla L.A., Christensen C.P., NiTZ A.J., UHL T.L., Electromyographic analysis of hip abductor exercises performed by a sample of total hip arthroplasty patients, J. Arthroplasty, 2009, 24, 1130-1136.

[11] Jiang N., Peng L., Al-Qwbani M., XIE G.P., Yang Q.M., ChaI Y., Zhang Q., YU B., Femoral version, neck-shaft angle, and acetabular anteversion in Chinese Han population: a retrospective analysis of 466 healthy adults, Medicine (Baltimore), 2015, 94, e891.

[12] Judd D.L., Dennis D.A., Thomas A.C., Wolfe P., DAYTon M.R., STEVENS-LAPSLEY J.E., Muscle strength and functional recovery during the first year after THA, Clin. Orthop. Relat. Res., 2014, 472, 654-664.

[13] Kopeć K., Kusz D., Sobota G., NowaK K., MierzwiŃSKi M., NowAK M., Gait analysis in patients after unilateral hip arthroplasty, Ortop. Traumatol. Rehabil., 2015, 17, 39-50.

[14] Ling S.M., Conwit R.A., TALbot L., Shermack M., WoOd J.E., Dredge E.M., Weeks M.J., Abernethy D.R., Metter E.J., Electromyographic patterns suggest changes in motor unit physiology associated with early osteoarthritis of the knee, Osteoarthritis Cartilage, 2007, 15, 1134-1140.
[15] Macadam P., Cronin J., Contreras B., An examination of gluteal muscle activity associated with dynamic hip abduction and hip external rotation exercise: a systematic review, Int. J. Sports Phys. Ther., 2015, 10, 573-591.

[16] Marshall A.R., Noronha M., Zacharias A., KaPAKOUlaKis T., GREEN R., Structure and function of the abductors in patients with hip osteoarthritis: Systematic review and meta-analysis, J. Back Musculoskelet. Rehabil., 2016, 29, 191-204.

[17] Maruyama M., Feinberg J.R., CAPello W.N., D’Antonio J.A., The Frank Stinchfield Award: Morphologic features of the acetabulum and femur: anteversion angle and implant positioning, Clin. Orthop. Relat. Res., 2001, 393, 52-65.

[18] MeEkins G.D., So Y., QuAN D., American Association of Neuromuscular and Electrodiagnostic Medicine evidencedbased review: use of surface electromyography in the diagnosis and study of neuromuscular disorders, Muscle Nerve, 2008, 38, 1219-1224.

[19] PAI V.S., Significance of the Trendelenburg test in total hip arthroplasty. Influence of lateral approaches, J. Arthroplasty, 1996, 11, 174-179.

[20] Reiman M.P., Bolgla L.A., Loudon J.K., A literature review of studies evaluating gluteus maximus and gluteus medius activation during rehabilitation exercises, Physiother. Theory Pract., 2012, 28, 257-268.

[21] Sims K.J., Richardson C.A., BraUer S.G., Investigation of hip abductor activation in subjects with clinical unilateral hip osteoarthritis, Ann. Rheum. Dis., 2002, 61, 687 $-692$.

[22] Stanisz A., Przystęny kurs statystyki z zastosowaniem STATISTICA PL na przykladach z medycyny, T. 2. Modele liniowe i nieliniowe, Statsoft Polska, 2007.

[23] Yang Y., Zuo J., LiU T., Xiao J., LiU S., GaO Z., Morphological Analysis of True Acetabulum in Hip Dysplasia (Crowe Classes I-IV) Via 3-D Implantation Simulation, J. Bone Joint Surg. Am., 2017, 6, e92. 